\title{
ESTUDO DA CONDUTIVIDADE HIDRÁULICA DE UM SOLO ALUVIAL EM LISÍMETROS ${ }^{1}$
}

\author{
Manoel Moises Ferreira de Queiroz $^{2}$, Bernd Rusteberg ${ }^{3}$, Hans Raj Gheyi ${ }^{4}$ \\ \& Hugo Orlando Carvallo Guerra ${ }^{4}$
}

\begin{abstract}
RESUMO
A condutividade hidráulica num solo Aluvial eutrófico, de textura franco arenosa, foi estudada em lisímetros $(1,25 \times 1,25 \times 1,25 \mathrm{~m})$ equipados com tensiômetros e tubos de acesso para sonda de neutrons, no Perímetro Irrigado de São Gonçalo, Sousa, PB. Após saturação do solo, os lisímetros foram cobertos com lona plástica para o ensaio de drenagem interna durante 15 dias, utilizando-se o método do perfil instantâneo. O ensaio teve continuidade por mais 13 dias, sem a lona plástica, aplicando-se o método do balanço hídrico com base no plano de fluxo nulo. Os resultados de $K(\theta)$ obtidos foram submetidos a ajustes matemáticos baseados na minimização do somatório dos desvios quadrados, através das funções exponencial, polinomial e da soma de duas exponenciais. A soma de duas exponenciais sem transformação logarítmica apresentou-se como a mais representativa dos resultados de $\mathrm{K}(\theta)$.
\end{abstract}

Palavras-chave: movimento de água, drenagem interna, balanço hídrico, perfil instantâneo

\section{STUDY OF HYDRAULIC CONDUCTIVITY OF AN ALLUVIAL SOIL IN LYSIMETERS}

\begin{abstract}
The hydraulic conductivity of an alluvial eutrophic sandy loam soil was determined in lysimeters $(1.25 \times 1.25 \times 1.25 \mathrm{~m})$ equipped with tensiometers and neutron probe access tube in the Irrigated Perimeter of São Gonçalo, Sousa-PB. After soil saturation, the lysimeters were covered with plastic sheets for study of internal drainage during 15 days, using the instantaneous profile method. The study was continued for another 13 days, without plastic sheet, using a water budget based on zero flux plan. The results of $K(\theta)$ obtained were submitted to mathematical adjustments based on the sum of least square of standard errors, by the exponential, polynomial and the sum of two exponential functions. The sum of two exponential without logarithmic transformation was found to be the most representative fitting mode for the $\mathrm{K}(\theta)$ results.
\end{abstract}

Key words: water movement, internal drainage, water budget, instantaneous profile

\footnotetext{
${ }^{1}$ Pesquisa financiada através de PDCT-NE Convênio CNPq - UFPB

${ }^{2}$ Professor Adjunto, Departamento de Engenharia Agrícola, Universidade Ouest de Maringá, Cascavel, PR. Email: moisesfq@sc.usp.br

${ }^{3}$ Institut fur Wasserbau der Universitat Hannover. D 3000 Hannover. R.F.A. Atualmente Professor Visitante na Escola de Engenharia Civil da Universidade Federal de Goiás. Convenio DAAD-CAPES. CEP 74650 - 220 Goiana, Go. E-mail: br@eec.ufg.br

${ }^{4}$ Professor Titular, Departamento de Engenharia Agrícola, Universidade Federal da Paraíba, CEP: 58109 - 090, Campina Grande, PB. Fone: (083) 310 1285, Fax: (083)310 1184.E-mails: hans@deag.ufpb.br; hugo@ deag.ufpb.br
} 


\section{INTRODUÇÃO}

A região semi-árida do Nordeste brasileiro, caracteriza-se por possuir aproximadamente $25 \%$ da superfície com problemas de salinização e/ou sodicidade (DNOCS, 1991), tornando-a improdutiva. Nesta região os processos de salinização geralmente se verificam em solos irrigados sem uma drenagem adequada. A água de irrigação traz um complemento de sais que se vão acumulando a medida em que as condições de drenagem são insuficientes para sua eliminação. Assim, o grau de salinização depende da quantidade de sais dissolvida na água. A concentração de sais encontrada na solução varia de menos de $0,0050 \%$ na água de chuva, a mais de $0,1 \%$ na água de irrigação até $1 \%$ na água de drenagem de solos moderadamente salinos, ou até 3\% em lixiviados de solos severamente salinizados (Prevedello, 1996). Quando a água se movimenta ela arrasta os solutos, pelo fluxo de massa, sendo que parte poderá ser adsorvida em outros locais, outra parte poderá ser absorvida pelas plantas, ou mesmo ser precipitada quando sua concentração excede sua solubilidade, como acontece na superfície do solo durante a evaporação (Prevedello, 1996).

Para aumentar a produção agrícola e melhorar o rendimento das culturas, no sentido de satisfazer a demanda crescente de alimentos e motivar a permanência do homem no campo, é imprescindível se conhecer e controlar os processos de salinização, prevendo-os e prevenindo-os na implantação de novas áreas irrigadas.

Considerando-se que a maioria dos problemas de salinização acontece em solos não saturados, a determinação da condutividade hidráulica do solo é de fundamental importância na resolução de problemas práticos ligados à irrigação, drenagem, transporte de nutrientes, entre outros (Bacchi \& Reichardt, 1988).

Existem diversos métodos para determinar a condutividade hidráulica em solos não saturados, subdivididos em métodos de campo (perfil instantâneo, camadas impermeáveis), de laboratório (drenagem interna, permeâmetros de carga constante e variável, ar quente) e empíricos, baseados na distribuição do tamanho dos poros (Prevedello, 1996). Embora demorado e trabalhoso, o método mais exato é o do perfil instantâneo (Hillel et al., 1972) por quantificar diretamente cada componente da equação de Richards (Pauletto et al., 1988) .

$\mathrm{O}$ presente trabalho constitui a fase inicial de um projeto global cuja finalidade era desenvolver e adaptar um modelo numérico de simulação de acumulação de sais (salinização) no solo. Assim, o objetivo deste trabalho foi determinar a condutividade hidráulica do solo em função da umidade [K( $(\theta)]$ e submeter os resultados a ajustamentos de modelos analíticos, visando escolher o melhor para suprir as necessidades de projetos de irrigação e drenagem e que, ao mesmo tempo, possa ser utilizado nos estudos de simulação de fluxo de água e transporte de sais no solo.

\section{MATERIAL E MÉTODOS}

O experimento foi conduzido no Perímetro Irrigado de São Gonçalo, Sousa, PB ( $\left.38^{\circ} 19^{\prime} \mathrm{W}, 6^{\circ} 50^{\prime} \mathrm{S}\right)$ em 3 tanques de alvenaria (lisímetros) de 1,25 x 1,25 x 1,25 m, cheios com um solo Aluvial eutrófico de textura franco-arenosa, retirado de uma parcela pertencente à Estação Experimental do Instituto Agronômico José Augusto Trindade (IAJAT/EMBRAPA). A Tabela 1 mostra as principais características do solo utilizado.
Tabela 1. Características físicas, químicas e mineralógicas do solo utilizado

\begin{tabular}{|c|c|}
\hline \multicolumn{2}{|l|}{ Granulometria $-\mathrm{g} \mathrm{kg}^{-1}$} \\
\hline Areia & 608,0 \\
\hline Silte & 315,0 \\
\hline Argila & 77,0 \\
\hline Classificação Textural & Franco Arenoso \\
\hline Densidade Aparente & 1,35 \\
\hline Densidade Real & 2,65 \\
\hline Porosidade Total - \% & 49,06 \\
\hline Umidade Natural $^{*}$ - $\mathrm{g} \mathrm{kg}^{-1}$ & 15,2 \\
\hline $33,4 \mathrm{kPa}$ & 157,5 \\
\hline $101,3 \mathrm{kPa}$ & 101,4 \\
\hline $202,6 \mathrm{kPa}$ & 83,9 \\
\hline $506,5 \mathrm{kPa}$ & 76,4 \\
\hline $1013,0 \mathrm{kPa}$ & 74,5 \\
\hline $1519,5 \mathrm{kPa}$ & 69,9 \\
\hline \multicolumn{2}{|l|}{ Complexo Sortivo $-\mathrm{cmol}_{\mathrm{c}} \mathrm{kg}^{-1}$} \\
\hline Cálcio & 6,50 \\
\hline Magnésio & 2,80 \\
\hline Potássio & 0,87 \\
\hline Sódio & 0,12 \\
\hline Bases Trocáveis & 10,29 \\
\hline Hidrogênio + Alumínio & 1,81 \\
\hline Capacidade de Troca Catiônica & 12,10 \\
\hline Carbono Orgânico - $\mathrm{g} \mathrm{kg}^{-1}$ & 9,7 \\
\hline Fósforo Assimilável - $\mathrm{mg} \mathrm{kg}^{-1}$ & 34,0 \\
\hline $\mathrm{pH}-\mathrm{H}_{2} \mathrm{O}(1: 2,5)$ & 6,50 \\
\hline Condutividade Elétrica - dS m ${ }^{-1}$ & 0,15 \\
\hline Minerologia $^{* *}$ & Ilita $>$ Caulinita $>$ Smectita \\
\hline
\end{tabular}

Devido ao grande número de fatores que influenciam o fenômeno de acumulação de sais no solo (infiltração lateral, variabilidade espacial do solo, volume de água aplicado) decidiuse realizar o estudo sob condições controladas de 3 lisímetros.

O processo de enchimento dos lisímetros e estabilização do solo foi demorado. Inicialmente, os lisímetros foram enchidos cuidadosamente com solo homogeneizado e durante três meses submetidos a irrigações periódicas; depois, foram submetidos ao processo natural de compactação, ficando expostos à intempérie durante mais cinco meses. Visando, ainda, promover uma estruturação do solo, plantou-se feijão (Phaseolus vulgaris L.) durante três meses incorporando-se a matéria verde ao solo antes da floração; finalmente, os lisímetros foram novamente expostos à intempérie, por mais quatro meses .

No centro de cada lisímetro instalou-se, verticalmente, um tubo de alumínio, para acesso da sonda de neutrons; no início fez-se uma avaliação da homogeneidade dos lisímetros, realizando-se medidas de umidade com sonda de neutrons a cada $10 \mathrm{~cm}$ de profundidade; posteriormente, foram feitas várias irrigações, até se conseguir a formação e manutenção de uma carga hidráulica de $5 \mathrm{~cm}$ sobre a superfície do solo. Nestas condições realizaram-se testes de infiltração e drenagem e uma primeira avaliação da condutividade hidráulica do solo, sob 
condições de saturação. Em cada um dos três lisímetros instalou-se uma bateria de tensiômetros, com manômetros de mercúrio, nas profundidades de 10,20,30,50,70,90 e $105 \mathrm{~cm}$ em torno do tubo de alumínio, num raio de $25 \mathrm{~cm}$. A forma do lisímetro e a tubulação de saída instalada permitiram manter um lençol freático permanente a $105 \mathrm{~cm}$ de profundidade.

$\mathrm{O}$ procedimento de enchimento dos lisímetros e detalhes dos ensaios de drenagem, inclusive, do comportamento hidrodinâmico, são dados por Gheyi (1989) e Queiroz (1990).

Em cada lisímetro, o solo foi saturado e coberto com lona plástica, a fim de evitar a evaporação. Nestas condições, procedeu-se ao ensaio de "Drenagem Interna" (Hillel et al., 1972) fazendo-se medições periódicas dos volumes de água drenados, e da umidade volumétrica através da sonda de neutrons e dos tensiômetros. Após 15 dias, retirou-se a lona plástica e continuaram-se as medições por mais 13 dias, com o solo em evaporação; conduzindo-se com estes dados um "Balanço Hídrico" (Royer \& Vachaud, 1974).

Os valores de condutividade hidráulica referentes à drenagem interna foram obtidos pelo método de Hillel et al. (1972) o que pressupõe a utilização da equação de Buckingham (1907):

$$
q=-K(\theta) \partial H / \partial z
$$

que, relacionada à equação da continuidade, conduz à equação diferencial geral dos fluxos de água, apresentada por Richards (1931):

$$
\partial \theta / \partial \mathrm{t}=\partial / \partial \mathrm{z}[\mathrm{K}(\theta) \partial \mathrm{H} / \partial \mathrm{z}]
$$

onde q é a densidade de fluxo; $K(\theta)$ a condutividade hidráulica do solo $\left(\mathrm{cm} \mathrm{dia}{ }^{-1}\right)$ em função de sua umidade $\theta\left(\mathrm{cm}^{3} \mathrm{~cm}^{-3}\right) ; \mathrm{H} \mathrm{o}$ potencial total da água no solo $(\mathrm{cm}) ; \mathrm{z}$ a coordenada vertical de posição $(\mathrm{cm})$ e t o tempo (dia).

Integrando a Eq. (2) em relação a z, da superfície do solo até a profundidade $L$, e explicitando $K(\theta)$ resulta:

$$
K(\theta)=\frac{\int_{0}^{L}(\partial \theta / \partial t) d z}{\partial H / \partial z}
$$

O gradiente $(\partial \theta / \partial \mathrm{t}) \mathrm{dz}$ foi obtido utilizando-se os dados de armazenamento de água $(\mathrm{S})$ calculados através do somatório dos perfis hídricos entre a superfície e o nível de referência $\mathrm{z}$ traçando-se a curva $S_{z}(t)$ - armazenamento de água numa profundidade $\mathrm{z}$ em função do tempo t. $\mathrm{O}$ fluxo que passa na profundidade $\mathrm{z}$, em um instante $\mathrm{t}$, é dado diretamente pela tangente da curva $\mathrm{S}_{\mathrm{z}}(\mathrm{t})$ no instante considerado (Vachaud et al., 1978). O $\partial \mathrm{H} / \partial \mathrm{z}$ foi avaliado através das curvas confeccionadas a partir dos dados de potenciais medidos com os tensiômetros.

$\mathrm{Na}$ fase de evaporação, a condutividade hidráulica também foi obtida pela Eq. (3) sendo que, neste caso, o valor de $(\partial \theta / \partial \mathrm{t}) \mathrm{dz}$ foi avaliado por meio de um "Balanço Hídrico", com base num plano de fluxo nulo que separa, dentro do perfil do solo, os fluxos ascendentes na parte superior (sob a ação da evaporação) dos fluxos descendentes na parte mais profunda (constituindo a drenagem). O nível onde o fluxo é nulo (plano de fluxo nulo) que corresponde a $\partial \mathrm{H} / \partial \mathrm{z}=0$, refere-se ao ponto de máximo da curva $\mathrm{H}(\mathrm{z})$. A posição desse plano é representado sobre os perfis de carga hidráulica e sua cota $\mathrm{Z}_{\mathrm{o}}$ evolui com o tempo (Royer \& Vachaud, 1974). A densidade de fluxo em um nível z de referência (acima de $\mathrm{Z}_{\mathrm{o}}$ ) foi calculada por meio do fluxo médio ascendente que passa através desta cota entre os tempos $\mathrm{t}_{1}$ e $\mathrm{t}_{2}$ (Eq. 4) considerando-se que o fluxo é nulo na cota $\mathrm{Z}_{\mathrm{o}}$, a qual representa a média entre duas profundidades sobre o plano.

$$
\mathrm{q}=\frac{[\partial \mathrm{S}]_{\mathrm{z}_{0}}^{\mathrm{z}}}{\mathrm{t}_{2}-\mathrm{t}_{1}}
$$

onde $\partial \mathrm{S}$ é a variação de armazenamento de água entre os níveis $\mathrm{Z}_{0}$ e Z, no intervalo de tempo considerado. O fluxo é relacionado a um valor médio de umidade obtido na seção $\mathrm{z}$ entre $\mathrm{t}_{1} \mathrm{e} \mathrm{t}_{2}, \mathrm{o}$ qual é reconduzido ao cálculo de $\mathrm{K}(\theta)$ ou do gradiente médio, obtido igualmente na seção $z$ entre $t_{1}$ e $t_{2}$. Os valores de $\theta$ e de $\partial \mathrm{H} / \partial \mathrm{Z}$ foram obtidos traçando-se as curvas $\theta(\mathrm{t})$ e $\mathrm{H}_{\mathrm{z}}(\mathrm{t})$ a partir das medições de umidade e de potencial.

A partir do conjunto de valores de condutividade hidráulica e de umidade de solo, procedeu-se ao ajustamento de modelos matemáticos, para se escolher o que melhor represente a relação $\mathrm{K}(\theta)$, com base na minimização do somatório dos desvios dos quadrados:

a) com transformação logarítmica de variáveis: foram testados os modelos exponencial e polinomial, a partir do ajustamento linear das variáveis transformadas;

b) sem transformação de variáveis: foram testados os modelos exponencial, polinomial, e a soma de duas exponenciais $[\mathrm{Y}=\mathrm{aEXP}(\mathrm{px})+\mathrm{bEXP}(\mathrm{qx})]$ por meio do método não paramétrico Simplex (Nelder \& Mead, 1965) utilizando o algoritmo desenvolvido por Cheviollote \& Touma (1987).

A condutividade hidráulica saturada foi calculada como parâmetro de ajustamento para comparação com os valores experimentais.

\section{RESULTADOS E DISCUSSÃO}

\section{Ensaios de drenagem interna (sem evaporação)}

Os três lisímetros apresentaram características físico-hídricas semelhantes (capacidade de armazenamento e infiltração de água). A Figura 1 mostra a drenagem acumulada em função do tempo para os três lisímetros. Constata-se que os lisímetros apresentaram comportamento hidrodinâmico idêntico, indicando que a compactação do solo foi quase uniforme, o que pode também ser confirmado ao se observar os valores das densidades globais obtidas por Queiroz (1990) pela sonda de raios gamma, a diferentes profundidades em cada lisímetro (densidades globais medias de 1,38; 1,41 e 1,42, para os lisímetros 1, 2 e 3, respectivamente). As Figuras $2 \mathrm{~A}$ e $2 \mathrm{~B}$ mostram as curvas de armazenamento de água em função do tempo para as camadas 0-30 e 30-60 cm durante o processo de drenagem, para os três lisímetros e permitem, também, estabelecer os valores de densidade de fluxo através da tangente à curva em pontos específicos do tempo. Os valores dos gradientes de carga para a profundidade de $25 \mathrm{~cm}$ foram obtidos a partir das diferenças de cargas, determinadas sobre as curvas $\mathrm{H}(\mathrm{t})$ a 20 e $30 \mathrm{~cm}$ de profundidade, nos referidos tempos da densidade de fluxo, como mostra a Figura 3A. Semelhante informação é encontrada na Figura 3B para a profundidade de $60 \mathrm{~cm}$. 


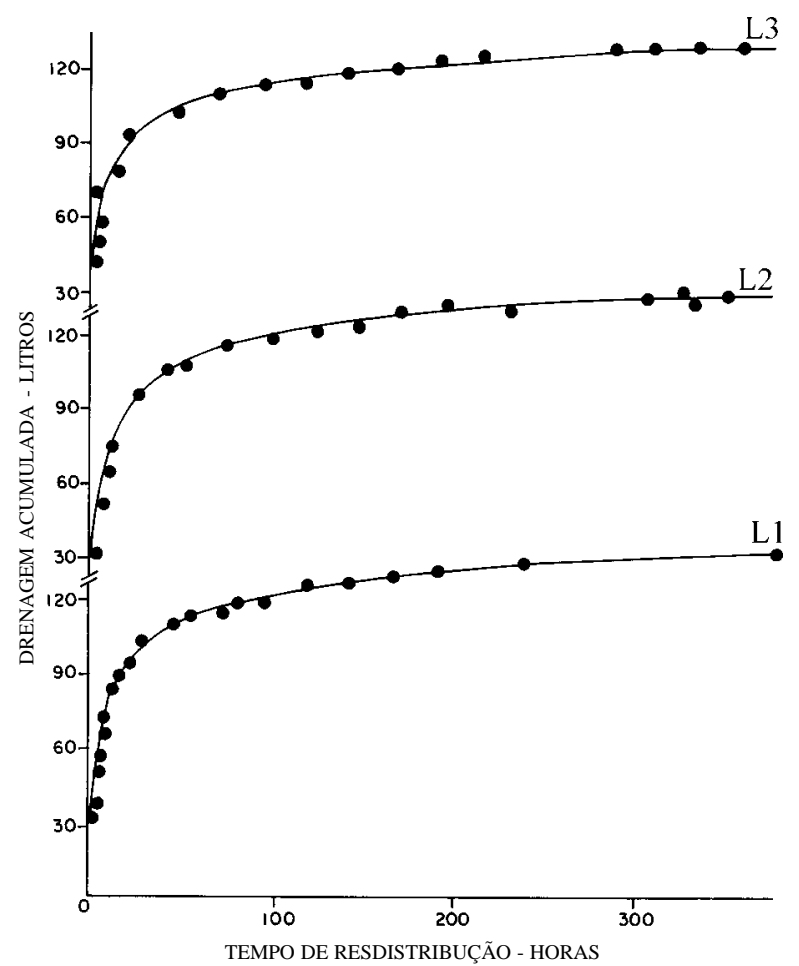

Figura 1. Drenagem acumulada em função do tempo para diferentes lisímetros $(\mathrm{L})$

A metodologia utilizada para a determinação da densidade de fluxo apresentou dificuldades, notadamente no início e no final da drenagem interna, pelo fato de que no início do ensaio (primeiras $2 \mathrm{~h}$ ) o processo da drenagem ocorreu rapidamente, com variações muito acentuadas de armazenamento de água e de potencial de carga, o que tornou difícil a definição das tangentes (Figuras 2A e 2B) e o estabelecimento das diferenças de cargas sobre as curvas $\mathrm{H}(\mathrm{t})$ nos tempos considerados (Figuras 3A e 3B). Após 200 horas, como a drenagem foi lenta, a variação de armazenamento de água foi muito pequena; por conseguinte, a definição das tangentes foi trabalhosa confundindo-se, às vezes, com a própria curva. $\mathrm{Na}$ faixa intermediária, entre 2 e $200 \mathrm{~h}$ de drenagem interna, os valores de densidade de fluxo e de gradiente de carga foram obtidos facilmente, mostrando-se mais precisos e seguros.

Esses resultados indicam que o método de drenagem interna pode ser utilizado na presença de um lençol freático próximo à superfície do solo, desde que a profundidades menores de $40 \mathrm{~cm}$ as medições sejam cuidadosamente realizadas e que o solo apresente características físicas semelhantes.

\section{Ensaio com evaporação (balanço hídrico)}

Apesar da utilização de um conjunto de 7 tensiômetros na fase com evaporação, foi difícil se definir a posição do plano de fluxo nulo e obter os gradientes de cargas, devido a evaporação não haver ultrapassado os $50 \mathrm{~cm}$ de profundidade de solo. Embora o cálculo das densidades de fluxos tenha sido facilitado pelo curto período evaporativo, foi possível determinar somente 4 valores de condutividade hidráulica $(0,014 ; 0,015 ; 0,035$ e $\left.0,05 \mathrm{~cm} \mathrm{dia}^{-1}\right)$ em uma faixa de variação de umidade restrita $(0,23$ a $0,25 \mathrm{~cm}^{3} \mathrm{~cm}^{-3}$ ), comprovando que o uso deste método para se obter mais resultados, além de necessitar de um número maior de tensiômetros, precisaria também aumentar o tempo do ensaio.

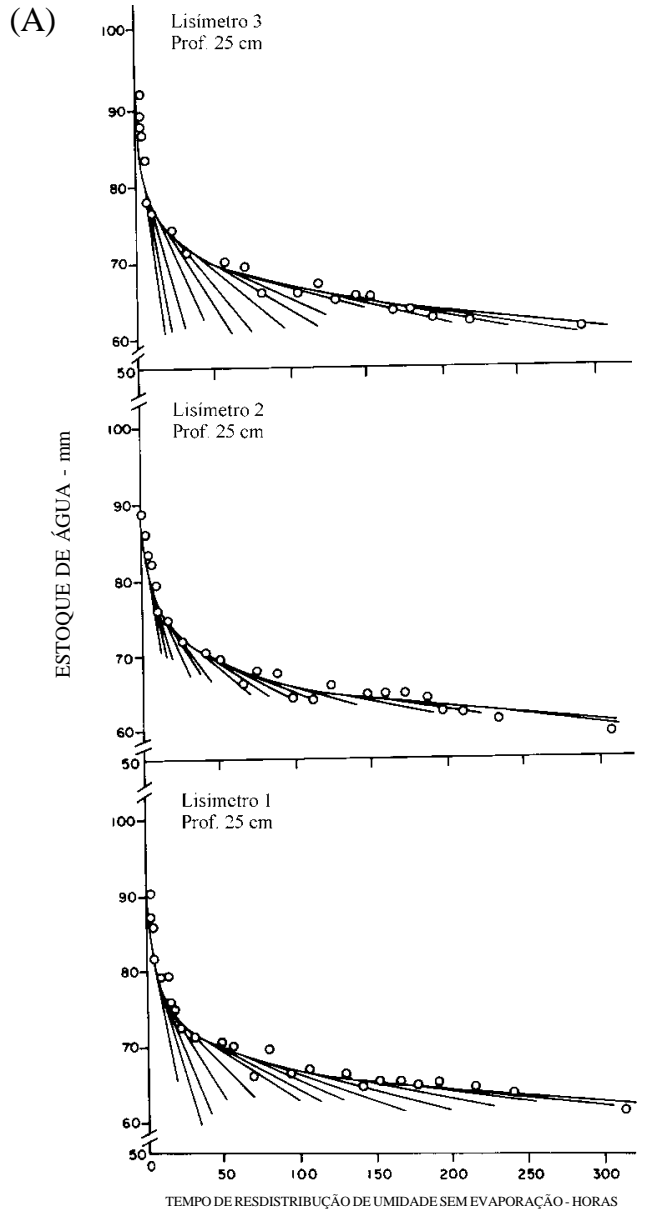

(B)

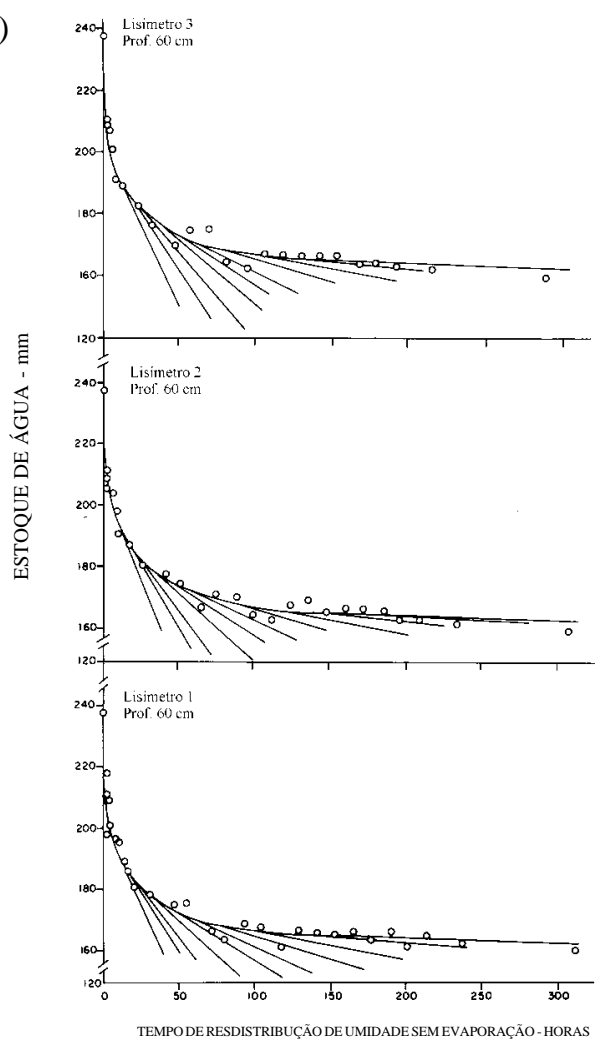

Figura 2. Curvas de armazenamento de água referentes à camada de (A) 25 e (B) $60 \mathrm{~cm}$ do solo, durante a drenagem interna, com tangente em pontos específicos do tempo para os três lisímetros utilizados 
(A)

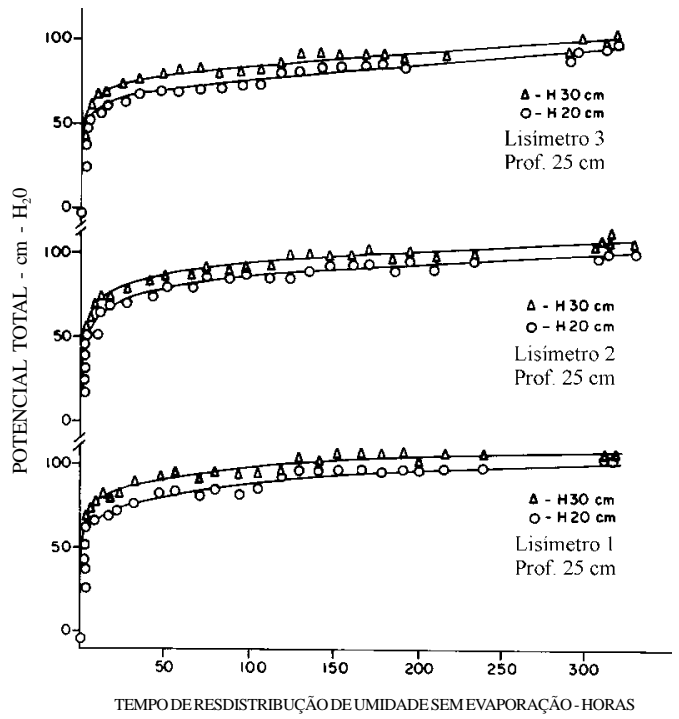

(B)

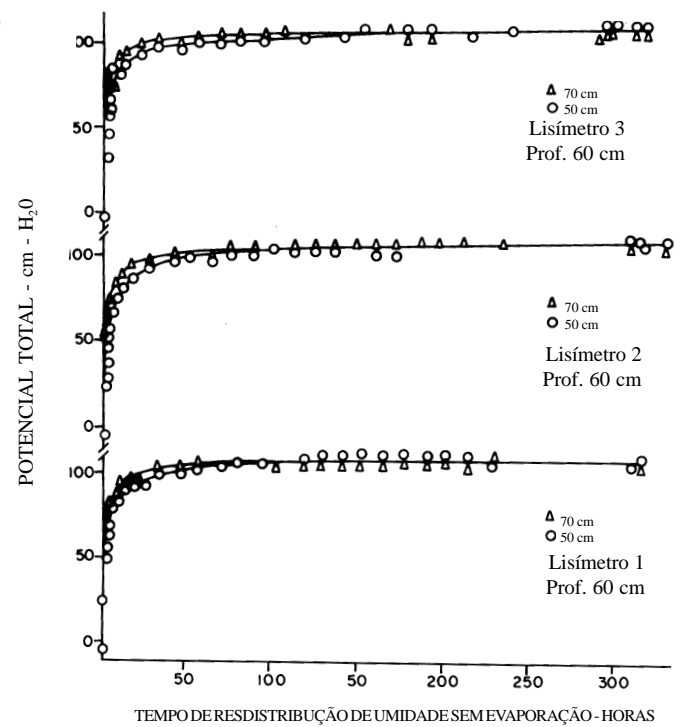

Figura 3. Evolução do potencial total de água no solo (A) 20/30 $\mathrm{cm}$ e (B) $50 / 70 \mathrm{~cm}$ de profundidade, durante a drenagem interna, para diferentes lisímetros

\section{Condutividade hidráulica}

A avaliação da condutividade hidráulica saturada $\left(\mathrm{K}_{\mathrm{o}}\right)$ se deu através do ensaio de infiltração, das curvas de drenagem acumulada (ambas realizadas na verificação da homogeneidade dos lisímetros), das curvas de drenagem interna e por meio de determinação em laboratório, utilizando-se amostras não deformadas de solo. Os valores obtidos se enquadram numa faixa de variação entre $60 \mathrm{e} 325 \mathrm{~cm} \mathrm{dia}^{-1} \mathrm{e}$ média de $172,4 \mathrm{~cm} \mathrm{dia}^{-1}$. Andrade et al. (1999) trabalhando com o mesmo solo e com ensaios de infiltração no campo obtiveram valores de condutividade hidráulica variando entre 8 e $131 \mathrm{~cm} \mathrm{dia}^{-1}$; já Barreto et al. (1998) utilizando o método do poço encontraram valores variando entre 20 e $171 \mathrm{~cm} \mathrm{dia}^{-1}$. Os maiores valores de condutividade hidráulica encontrados no presente trabalho são atribuídos ao fato dos solos dos lisímetros serem inicialmente disturbados, enquanto as determinações de Barreto et al. (1998) e Andrade et al. (1999) foram com amostras não deformadas.

A Figura 4 apresenta os valores de $K(\theta)$ para as profundidades de 25 e $60 \mathrm{~cm}$ para os três lisímetros, com e sem evaporação, confirmando a semelhança de comportamento entre os lisímetros. Observa-se que os resultados relativos à profundidade de $60 \mathrm{~cm}$, embora limitados a uma faixa restrita de umidade, se enquadraram bem na faixa de variação do conjunto de valores de $K(\theta)$ determinados para a profundidade de $25 \mathrm{~cm}$. Os resultados para a profundidade de $60 \mathrm{~cm}$ foram limitados a uma estreita faixa de variação de umidade, pois o solo nesta profundidade não secou completamente, devido a presença do lençol freático a $105 \mathrm{~cm}$ de profundidade. Embora com valores mais altos, os resultados estão de acordo com a bibliografia, observando-se aumento de $\mathrm{K}(\theta)$ com o conteúdo de água, até atingir valor constante, o qual corresponde á condutividade hidráulica sob condições de solo saturado (Carvallo et al., 1976).

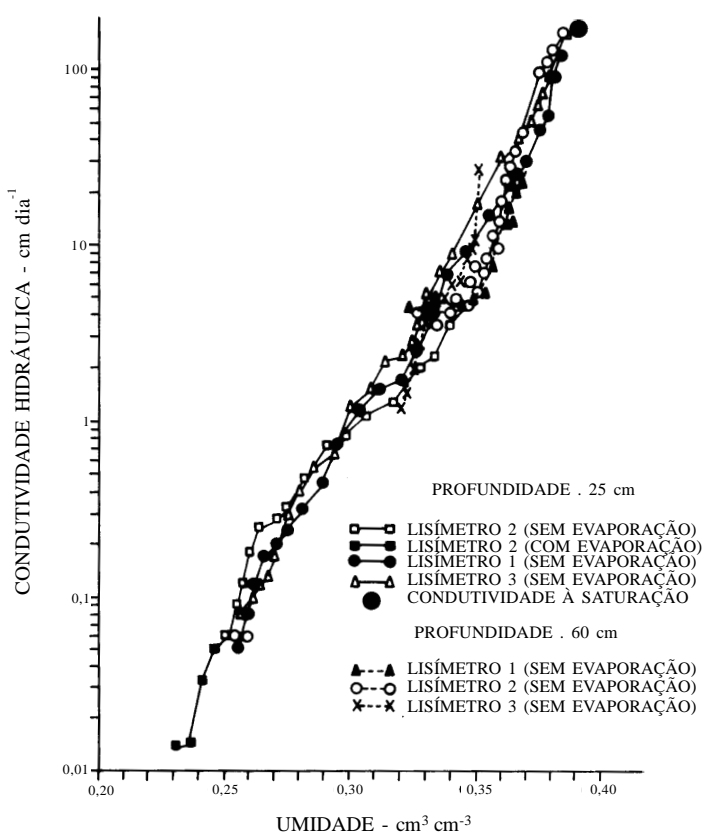

Figura 4. Curvas de condutividade hidráulica do solo em função da umidade média a 25 e $60 \mathrm{~cm}$ de profundidade, para diferentes lisímetros

\section{Ajustes matemáticos}

Com base na homogeneidade dos lisímetros, os ajustes matemáticos foram conduzidos sobre o conjunto total de dados, cujos resultados estão apresentados na Tabela 2 e na Figura 5.

Observa-se, nesta Tabela, que as funções com transformação logarítmica de variáveis forneceram excelentes coeficientes de determinação, $\mathrm{R}^{2}$, porém quando se volta para a variável inicial e se calcula a proporção de variância explicada, este coeficiente cai bruscamente, tanto para a função exponencial como para a polinomial. Os ajustes sem transformação logarítmica de variáveis forneceram valores de $\mathrm{R}^{2}$ próximo aos obtidos com os dados transformados; no entanto, a Figura 5 mostra que, com exceção do modelo que considera a soma de duas exponenciais, sem transformação logarítmica, os outros modelos subestimaram os valores de K quando o solo possui teores de umidades abaixo de $0,318 \mathrm{~cm}^{3} \mathrm{~cm}^{-3}$. O modelo que considera a soma de duas exponenciais, apesar de não apresentar o $\mathrm{R}^{2}$ mais alto, fornece um ajuste que, de fato, respeita a distribuição dos dados sobre a totalidade da faixa de variação de umidade, podendo ser considerado o mais satisfatório. $\mathrm{O}$ valor de $\mathrm{K}_{0}$ estimado por este modelo $\left(180 \mathrm{~cm} \mathrm{dia}^{-1}\right)$ enquadra-se muito bem dentro da faixa de valores de $\mathrm{K}_{0}$, observados experimentalmente no campo e no laboratório. 
Tabela 2. Funções de $K(\theta)$ obtidas através de ajustamentos matemáticos com e sem transformação logarítmica

\begin{tabular}{|c|c|}
\hline \multicolumn{2}{|l|}{ A. Com transformação logarítmica } \\
\hline \multicolumn{2}{|c|}{ Exponencial } \\
\hline \multicolumn{2}{|l|}{ Ajustamento linear: } \\
\hline $\log K(\theta)=-7,1866+23,333 \theta$ & $\mathrm{R}^{2}=0,980^{* *}$ \\
\hline \multicolumn{2}{|l|}{ Variável inicial: } \\
\hline$K(\theta)=0,650710^{-7} \cdot \operatorname{EXP}(54,717 \theta)$ & $\mathrm{R}^{2}=0,779^{* *}$ \\
\hline \multicolumn{2}{|c|}{ Polinomial } \\
\hline \multicolumn{2}{|l|}{ Ajustamento linear: } \\
\hline $\log K(\theta)=8,8339+16,844 \log \theta$ & $\mathrm{R}^{2}=0,973^{* *}$ \\
\hline \multicolumn{2}{|l|}{ Variável inicial: } \\
\hline$K(\theta)=0,6821810^{9} \theta^{16,844}$ & $\mathrm{R}^{2}=0,662^{* *}$ \\
\hline B. Sem transformação logarítmica & \\
\hline
\end{tabular}

Exponencial

$\mathrm{K}(\theta)=210^{-10} . \operatorname{EXP}(70,575 \theta)$

Polinomial

$\mathrm{K}(\theta)=0,9210^{13} \theta^{26,003}$

$\mathrm{R}^{2}=0,945^{* *}$

$\mathrm{K}_{0}=207 \mathrm{~cm} \mathrm{dia}^{-1}$

Soma de duas exponenciais

$\mathrm{K}(\theta)=0,215810^{-8} . \operatorname{EXP}(23,025 \theta)$

$\mathrm{R}^{2}=0,922^{* *}$

$+0,6571610^{-8} \cdot \operatorname{EXP}(61,784 \theta)$

$\mathrm{K}_{0}=180 \mathrm{~cm} \mathrm{dia}^{-1}$

** Significativo a nível de $1 \%$ de probabilidade

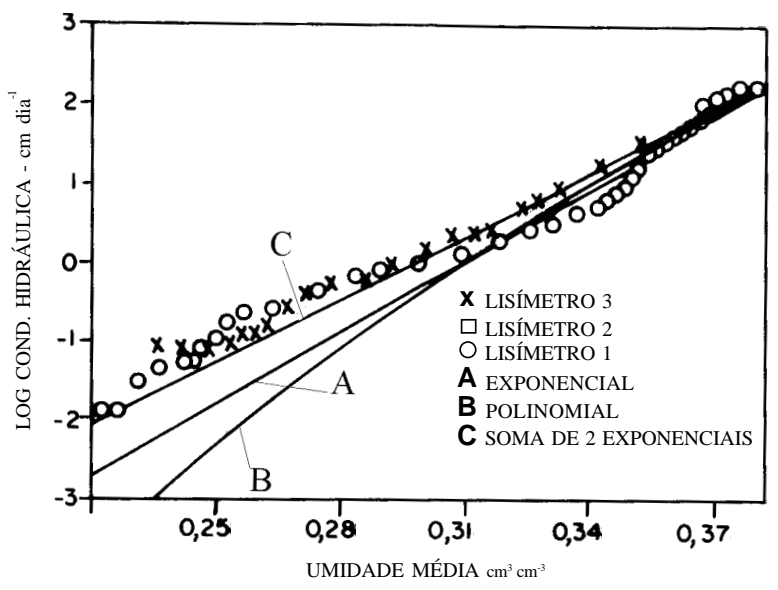

Figura 5. Curvas de ajustamento para relação entre a condutividade hidráulica e teor de umidade do solo a $25 \mathrm{~cm}$ de profundidade, para diferentes lisímetros. (A) Exponencial, (B) Polinomial e (C) Soma de duas exponenciais

\section{CONCLUSÕES}

1. O método da "drenagem interna" funcionou satisfatoriamente na obtenção da condutividade hidráulica do solo, em função do teor de umidade.

2. O método do balanço hídrico permitiu a determinação da condutividade hidráulica $K(\theta)$ para valores mais baixos de umidade de solo que aqueles obtidos com o método da drenagem interna.

3. Dos modelos utilizados, a soma de duas exponenciais sem transformação logarítmica de variáveis, foi o que melhor se ajustou aos dados, sendo representativo em todo o conjunto de valores de $\mathrm{K}(\boldsymbol{\theta})$. Os outros modelos apresentaram desvios que tornam os ajustes inadequados em faixas de umidade menor que $0,318 \mathrm{~cm}^{3} \mathrm{~cm}^{-3}$.

4. O modelo envolvendo a soma de duas exponenciais é recomendado na elaboração de projetos de irrigação e drenagem e para estudos de simulação do fluxo de água e de sais no solo.

\section{REFERÊNCIAS BIBLIOGRÁFICAS}

ANDRADE, A.R.; CARVALLO, H.O.; BARRETO, A.; LIMA, C.A.; FILGUEIRA, H. Variabilidade espacial da condutividade hidráulica saturada num solo aluvial do trópico semi-árido. In: CONGRESSO CHILENO DE INGENIERIA AGRÍCOLA, 3. Chillán, Chile. Memória. 1999. 6p.

BACCHI, O.O.S.; REICHARDT, K. Escalonamento de propriedades hídricas na avaliação de métodos de determinação da condutividade hidráulica de solos. Revista Brasileira de Ciência do Solo, Campinas, v. 12, p. 217-223. 1988.

BARRETO, A.N.; BEZERRA, J.R.; AZEVEDO, C.A.; DANTAS, J. Variabilidade espacial da condutividade hidráulica saturada num solo aluvional da Estação Experimental de São Gonçalo, PB. CONGRESSO BRASILEIRO DE ENGENHARIA AGRÍCOLA, 27. Poços de Caldas. Anais... Poços de Caldas: SBEA/UFLA. Junho, 1998. 3p.

BUCKINGHAM, E. Studies on the movement of soil moisture [S.1.], 1907.61p. USDA Bureau, Soils Bulletin 38

CARVALLO, H. O.; CASSEL, D.K.; HAMMOND, J.; BAUER, A. Spatial variability of in situ unsaturated hydraulic conductivity of Maddock sandy loam. Soil Science, v. 121, p.1-8. 1976.

CHEVIOLLOTE, H.; TOUMA, J. Optimum logical optimisation de paramétres non linéaires. Daker: ORSTOM, 1987. 19p.

DNOCS (Fortaleza, CE). Relatório técnico da Missão Húngara. Fortaleza, 1991. (não paginado)

GHEYI, H.R. Modelo numérico para previsão de riscos de salinização em áreas irrigadas. Campina Grande:UFPB. 1989. 72p. (Relatório Anual do Projeto PB-42. Convênio PDCT - CNPq/BID).

HILLEL, D.; KRENTOS, V.D.; STYLLIANOU, Y. Procedure and test of an internal drainage method for measuring hydraulic characteristics "in situ". Soil Science, v. 144, p. 395-400, 1972.

NELDER, J.A.; MEAD, R. A simplex method for function minimization. Computer Journal. 308-313, 1965.

PAULETTO, E.A.; LIBARDI, P.L.; MANFRON, P.A.; MORAES, S.O. Determinação da condutividade hidráulica de solos a partir da curva de retenção de água. Revista Brasileira de Ciência de Solo. Campinas, SP. v. 12, p. 189-195, 1988.

PREVEDELLO, C.L. Fisica do solo. Com problemas resolvidos. Curitiba. 446 p. 1996.

QUEIROZ, M.M.F. de. Estudo da condutividade hidráulica de um solo Aluvial franco- arenoso em experimento de lisímetro. Campina Grande: UFPB, 1990, 136p. Dissertação Mestrado

RICHARDS, L.A. Capillary conduction of liquids through porous medium. Physics, v. 1.p. 318-333, 1931.

ROYER, J.M.; VACHAUD, G. Determination directe de l'infiltration par mesure des teneurs en eau et des succions. Hydrological Sciences Bulletin, v. 19, p. 319-336, 1974.

VACHAUD, G.; DANCETTE, C.; SONKO, S.; THONY, J.L. Methodes de caractérisation hydrodynamique "in situ"d'un solo non saruré. Application à deux types de sol du Sénegal en vue de la détermination des termes du bilan hydrique. Annales Agronomiques, Paris, v. 29, p. 1-36, 1978. 